\title{
Magnetic Moments of the Octet Baryons in a Chiral Quark Potential Model
}

\author{
Georg Wagner, Alfons J. Buchmann, and Amand Faessler \\ Institute for Theoretical Physics, University of Tübingen, Auf der Morgenstelle 14, \\ D-72076 Tübingen, Germany
}

\begin{abstract}
In quark potential models, two-body current contributions to baryon magnetic moments arise necessarily to satisfy the continuity equation for the electromagnetic current. On the other hand, the naïve additive quark model predicts the experimental octet magnetic moments to within 5\%. We demonstrate that consistently derived two-body current contributions to the octet baryon magnetic moments are individually large, but tend to cancel each other globally.
\end{abstract}

PACS: $\quad$ 12.39.Pn $\quad$ Potential models

13.40.Gp Electromagnetic Form Factors

13.40.Em Electric and magnetic moments

Keywords: Quark Potential Models, Electromagnetic Two-body Currents, Continuity Equation, Octet Baryon Magnetic Moments and Form Factors, Chiral Symmetry

\footnotetext{
${ }^{\dagger}$ supported by the BMBF under contract number 06Tü746(2) and by the DFG Graduiertenkolleg MU705/3.
} 
While the importance of two-body currents for electromagnetic properties of nuclei is clearly established [1], the effect of two-body currents inside baryons is not very well understood (see ref.[2] and refs. therein). In this respect, the striking agreement of the naïve constituent quark model predictions for the octet baryon magnetic moments (summing up the magnetic moments of three free quarks gives results as close as $5 \%$ to the experimental values [3, 4]) is quite surprising, and probably has hampered a systematic study of exchange currents in the baryons for some time.

In some previous works two-body exchange current contributions to electromagnetic observables of the non-strange baryons [2] and the deuteron [5] have been studied in detail. The description of the full baryon octet involves the extension to non-equal quark masses in the Hamiltonian, the electromagnetic current operators and the baryon wave functions.

In the chiral quark potential model $(\chi \mathrm{QPM})$, a baryon is described as a nonrelativistic system of three constituent quarks. The quark dynamics is governed by two-body potentials. These potentials are motivated by the main properties of QCD [6] and some experimental facts, namely asymptotic freedom at short distances, chiral symmetry and its dynamical breaking at intermediate distances, and confinement at large distances. The spontaneous breaking of chiral symmetry is responsible for the constituent quark mass generation, as well as for the appearence of the pseudoscalar Goldstone pions or the pseudoscalar nonet in the case of three quark flavors. In the chiral quark potential model, this is modeled by a one-pion exchange potential between constituent quarks [7], together with the exchange potential of its chiral partner, the scalar-isoscalar sigma meson [8]".

The $\chi$ QPM Hamiltonian in the case of three non-equal quark masses $m_{i}$ is written as

$$
H=\sum_{i=1}^{3}\left(m_{i}+\frac{\mathbf{p}_{i}^{2}}{2 m_{i}}\right)-\frac{\mathbf{P}^{2}}{2\left(m_{1}+m_{2}+m_{3}\right)}+\sum_{i<j}^{3} V^{\operatorname{Conf}}\left(\mathbf{r}_{i}, \mathbf{r}_{j}\right)+\sum_{i<j}^{3} V^{\operatorname{Res}}\left(\mathbf{r}_{i}, \mathbf{r}_{j}\right) .
$$

The confinement potential is given by $\left(\boldsymbol{\lambda}_{i}\right.$ are the Gell-Mann SU(3) color matrices)

$$
V^{\operatorname{Conf}}\left(\mathbf{r}_{i}, \mathbf{r}_{j}\right)=-a_{c} \boldsymbol{\lambda}_{i} \cdot \boldsymbol{\lambda}_{j}\left(\mathbf{r}_{i}-\mathbf{r}_{j}\right)^{2} .
$$

\footnotetext{
${ }^{1}$ For a consistent treatment of chiral symmetry breaking one should include the exchange of the whole pseudoscalar nonet. In the present work, we neglect the Kaon and $\eta$-exchanges due to their higher mass.
} 
The residual interactions comprise the chiral interactions at intermediate and the one-gluon exchange at short range. The nonrelativistic one-pion and one-sigma exchange potentials are:

$$
\begin{aligned}
V^{O P E P}\left(\mathbf{r}_{i}, \mathbf{r}_{j}\right) & =\frac{g_{\pi q}^{2}}{4 \pi} \frac{\Lambda_{\pi}^{2}}{\Lambda_{\pi}^{2}-\mu^{2}} \frac{\boldsymbol{\tau}_{i} \cdot \boldsymbol{\tau}_{j}}{4 m_{i} m_{j}} \boldsymbol{\sigma}_{i} \cdot \nabla_{r} \boldsymbol{\sigma}_{j} \cdot \nabla_{r}\left(\frac{e^{-\mu r}}{r}-\frac{e^{-\Lambda_{\pi} r}}{r}\right) \\
V^{O S E P}\left(\mathbf{r}_{i}, \mathbf{r}_{j}\right) & =-\frac{g_{\sigma q}^{2}}{4 \pi} \frac{\Lambda_{\sigma}^{2}}{\Lambda_{\sigma}^{2}-m_{\sigma}^{2}}\left(\frac{e^{-m_{\sigma} r}}{r}-\frac{e^{-\Lambda_{\sigma} r}}{r}\right)
\end{aligned}
$$

where $r=|\mathbf{r}|=\left|\mathbf{r}_{i}-\mathbf{r}_{j}\right|$. Here $\boldsymbol{\sigma}_{i}$ and $\boldsymbol{\tau}_{i}$ denote the spin and isospin of the i-th quark, respectively. According to the chiral symmetry arguments in ref. [8] the parameters of the $\pi-($ coupling only to $\mathrm{u}-$ and $\mathrm{d}-$-quarks) and the flavor-blind $\sigma$-meson are simultaneously fixed:

$$
\frac{g_{\sigma q}^{2}}{4 \pi}=\frac{g_{\pi q}^{2}}{4 \pi}=\left(\frac{3}{5} \frac{m_{u}}{m_{N}}\right)^{2} \frac{g_{\pi N}^{2}}{4 \pi} \quad, \quad m_{\sigma}^{2}=\left(2 m_{u}\right)^{2}+\mu^{2} \quad, \quad \Lambda_{\pi}=\Lambda_{\sigma} \equiv \Lambda .
$$

We use $g_{\pi N}^{2} / 4 \pi=13.845$ and the pion mass $\mu=138 \mathrm{MeV}$. The cut-off $\Lambda$ describes the range of the meson-quark interaction region, due to the finite sizes of the constituent quark and the mesons. The one-gluon exchange potential is used in the well known form of [9]:

$$
\begin{aligned}
V^{O G E P}\left(\mathbf{r}_{i}, \mathbf{r}_{j}\right) & =\frac{\alpha_{s}}{4} \boldsymbol{\lambda}_{i} \cdot \boldsymbol{\lambda}_{j}\left\{\frac{1}{r}-\frac{\pi}{2}\left(\frac{1}{m_{i}^{2}}+\frac{1}{m_{j}^{2}}+\frac{4}{3} \frac{\boldsymbol{\sigma}_{i} \cdot \boldsymbol{\sigma}_{j}}{m_{i} m_{j}}\right) \delta(\mathbf{r})-\frac{1}{4 m_{i} m_{j}}\left(3 \boldsymbol{\sigma}_{i} \cdot \hat{\mathbf{r}} \boldsymbol{\sigma}_{j} \cdot \hat{\mathbf{r}}-\boldsymbol{\sigma}_{i} \cdot \boldsymbol{\sigma}_{j}\right) \frac{1}{r^{3}}\right. \\
& \left.-\frac{1}{8 r^{3}}\left(3\left(\mathbf{r} \times\left(\frac{\mathbf{p}_{i}}{m_{i}}-\frac{\mathbf{p}_{j}}{m_{j}}\right)\right) \cdot\left(\frac{\boldsymbol{\sigma}_{i}}{m_{i}}+\frac{\boldsymbol{\sigma}_{j}}{m_{j}}\right)-\left(\mathbf{r} \times\left(\frac{\mathbf{p}_{i}}{m_{i}}+\frac{\mathbf{p}_{j}}{m_{j}}\right)\right) \cdot\left(\frac{\boldsymbol{\sigma}_{i}}{m_{i}}-\frac{\boldsymbol{\sigma}_{j}}{m_{j}}\right)\right)\right\} .
\end{aligned}
$$

For the baryon wave functions, we use simple $(0 s)^{3}$ harmonic oscillator states. The harmonic oscillator lengthes $b_{\boldsymbol{\rho}}$ and ${ }^{b} \boldsymbol{\lambda}$ in the directions of the internal Jacobi-coordinates $\boldsymbol{\rho}=\left(\mathbf{r}_{1}-\mathbf{r}_{2}\right)$ and $\boldsymbol{\lambda}=\mathbf{r}_{3}+\left(m_{1} \mathbf{r}_{1}+m_{2} \mathbf{r}_{2}\right) /\left(m_{1}+m_{2}\right)$ can be expressed in terms of quark mass ratios and the oscillator length $b_{N}$ used for the nucleon and the $\Delta$-resonance. The orbital part of the wave function $\Phi_{3 q}\left(f_{1} f_{2} f_{3}, \boldsymbol{\rho}, \boldsymbol{\lambda}\right)$ depends on the 3 quark flavors $f_{1}, f_{2}, f_{3}$, and the full wave function can be written as

$$
|B\rangle=\frac{1}{\sqrt{2}} \sum_{S_{12}=0,1}\left(\sum_{f_{1} f_{2} f_{3}}{ }^{B} C^{S_{12}}\left(f_{1} f_{2} f_{3}\right) \times \Phi_{3 q}\left(f_{1} f_{2} f_{3}, \boldsymbol{\rho}, \boldsymbol{\lambda}\right) \times\left|f_{1} f_{2} f_{3}\right\rangle\right) \times\left|S, S_{z} ; S_{12}\right\rangle \times\left|\square_{C}\right\rangle .
$$

$\left|f_{1} f_{2} f_{3}\right\rangle$ is the flavor part of the wave function, and the antisymmetry lies in the color space. The coefficients ${ }^{B} C^{S_{12}}\left(f_{1} f_{2} f_{3}\right)$ are SU(3) Clebsch-Gordan coefficients. These wave functions 
can be found for example in [4]. We use a different overall sign for the mixed symmetric states than in [1].

In this form the $\chi \mathrm{QPM}$ has been used to calculate the nucleon and $\Delta$ spectrum (positive parity), and has also been applied to the NN system with a fair amount of success [8, 10, 11]. As usual, the parameters for the present calculation are fitted to the nucleon and $\Delta$-resonance masses.

table 1

The baryon masses $m_{B}$ are determined as the expectation values of Hamiltonian (1) for the wave functions (7). The result is shown in table 2 .

Gauge invariance is the key requirement when calculating electromagnetic properties of the baryons. Any isospin- or momentum-dependent two-body potential in the Hamiltonian gives rise to additional two-body exchange current contributions to the total electromagnetic current. These two-body currents are necessary in order to satisfy the continuity equation for the electromagnetic current. Here, we list the electromagnetic currents, that have to be considered for consistency with our Hamiltonian of eq.(11). They have been derived in a number of papers [2, 5] for the case of non-strange quarks.

The nonrelativistic one-body current of the quarks of figure 1(a), referred to as impulse approximation, is given by

$$
\mathbf{J}_{i m p}\left(\mathbf{r}_{i}, \mathbf{q}\right)=\frac{e_{i}}{2 m_{i}}\left(i\left[\boldsymbol{\sigma}_{i} \times \mathbf{p}_{i}, e^{i \mathbf{q} \cdot \mathbf{r}_{i}}\right]+\left\{\mathbf{p}_{i}, e^{i \mathbf{q} \cdot \mathbf{r}_{i}}\right\}\right)
$$

In the $\mathrm{SU}(3)$-flavor case the charge operator for pointlike quarks is

$$
e_{i}=\frac{e}{2}\left(B_{i}+S_{i}+\boldsymbol{\tau}_{z}^{(i)}\right)
$$

where $B_{i}=1 / 3$ is the baryon number and $S_{i}$ is the strangeness quantum number of the $\mathrm{i}-$ th quark. Within the picture of dressed quasi-particle constituent quarks it is necessary to introduce a finite electromagnetic size of the quarks [2]. Motivated by the vector meson dominance picture [12], a possible parametrization for the electromagnetic quark size is to 
multiply the expression for the quark charge by a simple monopole form. The electromagnetic constituent quark size $r_{\gamma q}^{2}=0.36 \mathrm{fm}^{2}$ (independent of $\mathrm{SU}(3)$-flavor) is related to the vectormeson mass, and is taken from the calculation of the charge radii of the nucleons [2]. We multiply the expressions for the current operators by the electromagnetic quark form factor

$$
F_{\gamma q}\left(\mathbf{q}^{2}\right)=\left(1+\frac{\mathbf{q}^{2} r_{\gamma q}^{2}}{6}\right)^{-1}
$$

For the pion, due to its isospin structure, we get the well known pion-pair $(\pi q \bar{q})$ current, figure 1(b), and the isovector pionic $(\gamma \pi \pi)$ current, figure $1(\mathrm{c})$ :

$$
\begin{aligned}
& \mathbf{J}_{\pi q \bar{q}}^{I S}\left(\mathbf{r}_{i}, \mathbf{r}_{j}, \mathbf{q}\right)=\frac{i e}{6} \frac{g_{\pi q}^{2}}{4 \pi} \frac{\Lambda^{2}}{\Lambda^{2}-\mu^{2}}\left\{\frac{\boldsymbol{\tau}_{i} \cdot \boldsymbol{\tau}_{j}}{8 m_{i}^{3} m_{j}} e^{i \mathbf{q} \cdot \mathbf{r}_{i}} \mathbf{q} \times \nabla_{\mathbf{r}} \boldsymbol{\sigma}_{j} \cdot \nabla_{\mathbf{r}}\left(\frac{e^{-\mu r}}{r}-\frac{e^{-\Lambda r}}{r}\right)+(i \leftrightarrow j)\right\} \\
& \mathbf{J}_{\pi q \bar{q}}^{I V}\left(\mathbf{r}_{i}, \mathbf{r}_{j}, \mathbf{q}\right)=e \frac{g_{\pi q}^{2}}{4 \pi} \frac{\Lambda^{2}}{\Lambda^{2}-\mu^{2}}\left\{\frac{\left(\boldsymbol{\tau}_{i} \times \boldsymbol{\tau}_{j}\right)_{3}}{4 m_{i} m_{j}} e^{i \mathbf{q} \cdot \mathbf{r}_{i}} \boldsymbol{\sigma}_{i} \boldsymbol{\sigma}_{j} \cdot \nabla_{\mathbf{r}}+(i \leftrightarrow j)\right\}\left(\frac{e^{-\mu r}}{r}-\frac{e^{-\Lambda r}}{r}\right) \\
& \mathbf{J}_{\gamma \pi \pi}^{I V}\left(\mathbf{r}_{i}, \mathbf{r}_{j}, \mathbf{q}\right)=e \frac{g_{\pi q}^{2}}{4 \pi} \frac{\Lambda^{2}}{\Lambda^{2}-\mu^{2}} \frac{\left(\boldsymbol{\tau}_{i} \times \boldsymbol{\tau}_{j}\right)_{3}}{4 m_{i} m_{j}} \boldsymbol{\sigma}_{i} \cdot \nabla_{i} \boldsymbol{\sigma}_{j} \cdot \nabla_{j} \int_{-1 / 2}^{1 / 2} d v e^{i \mathbf{q} \cdot(\mathbf{R}-\mathbf{r} v)}\left(\mathbf{z}_{\mu} \frac{e^{-L_{\mu} r}}{L_{\mu} r}-\mathbf{z}_{\Lambda} \frac{e^{-L_{\Lambda} r}}{L_{\Lambda} r}\right) .
\end{aligned}
$$

For the pionic current we have used the abbreviations $\mathbf{R}=\left(\mathbf{r}_{i}+\mathbf{r}_{j}\right) / 2, \mathbf{z}_{m}(\mathbf{q}, \mathbf{r})=L_{m} \mathbf{r}+$ ivrq, and $L_{m}(q, v)=\left[\frac{1}{4} q^{2}\left(1-4 v^{2}\right)+m^{2}\right]^{1 / 2}$. We use the same monopole form factor of eq.(10) for the extended photon-pion vertex as for the photon-quark vertex.

The gluon-pair current of figure $1(\mathrm{~d})$ is given by

$$
\mathbf{J}_{g q \bar{q}}\left(\mathbf{r}_{i}, \mathbf{r}_{j}, \mathbf{q}\right)=-\frac{\alpha_{s}}{8} \boldsymbol{\lambda}_{i} \cdot \boldsymbol{\lambda}_{j}\left\{\frac{e_{i}}{m_{i}} e^{i \mathbf{q} \cdot \mathbf{r}_{i}}\left(\frac{\boldsymbol{\sigma}_{i}}{m_{i}}+\frac{\boldsymbol{\sigma}_{j}}{m_{j}}\right) \times \mathbf{r}+(i \leftrightarrow j)\right\} \frac{1}{r^{3}} .
$$

The gluon-pair current is related to the spin-orbit part in eq.(6). As discussed in [2], one should then for consistency also consider two-body currents corresponding to the scalar

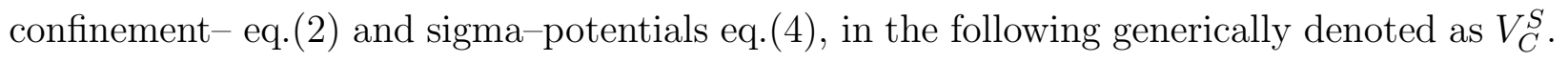
Including the lowest order $\mathcal{O}\left(\frac{1}{m_{q}^{2}}\right)$ relativistic corrections leads to the following structure of the scalar exchange potential:

$$
\begin{aligned}
V^{S}= & V_{C}^{S}-\frac{1}{4}\left(\left\{\left(\frac{\boldsymbol{\sigma}_{i} \cdot \mathbf{p}_{i}}{m_{i}}\right)^{2}, V_{C}^{S}\right\}+\left\{\left(\frac{\boldsymbol{\sigma}_{j} \cdot \mathbf{p}_{j}}{m_{j}}\right)^{2}, V_{C}^{S}\right\}+\frac{1}{2}\left(\frac{\boldsymbol{\nabla}_{i}^{2}}{m_{i}^{2}}+\frac{\boldsymbol{\nabla}_{j}^{2}}{m_{j}^{2}}\right) V_{C}^{S}\right. \\
& \left.+\frac{1}{r} \frac{\mathrm{d} V_{C}^{S}}{\mathrm{~d} r}\left[\frac{1}{2}\left(\frac{\boldsymbol{\sigma}_{i}}{m_{i}}+\frac{\boldsymbol{\sigma}_{j}}{m_{j}}\right) \cdot \mathbf{r} \times\left(\frac{\mathbf{p}_{i}}{m_{i}}-\frac{\mathbf{p}_{j}}{m_{j}}\right)+\frac{1}{2}\left(\frac{\boldsymbol{\sigma}_{i}}{m_{i}}-\frac{\boldsymbol{\sigma}_{j}}{m_{j}}\right) \cdot \mathbf{r} \times\left(\frac{\mathbf{p}_{i}}{m_{i}}+\frac{\mathbf{p}_{j}}{m_{j}}\right)\right]\right) .
\end{aligned}
$$

Also from the baryon mass spectroscopy and the NN phase shift analysis, these spin-orbit terms in the scalar potentials seem to be needed [11, 13]. Similarily to the gluon case, they 
provide by minimal substitution a two-body confinement and sigma-pair current, see figure 1(e). This current can be expressed by use of $V^{\text {Conf }}$ of eq.(2) as:

$$
\mathbf{J}_{\text {conf }}\left(\mathbf{r}_{i}, \mathbf{r}_{j}, \mathbf{q}\right)=-\left\{\frac{e_{i}}{2 m_{i}^{2}} e^{i \mathbf{q} \cdot \mathbf{r}_{i}} V^{C o n f} i \boldsymbol{\sigma}_{i} \times \mathbf{q}+(i \leftrightarrow j)\right\}
$$

By analogy [2], we have to replace $V^{\text {Conf }}$ in (14) by the potential $V^{O S E P}$ of eq.(1) to obtain the sigma-pair current.

Calculating the magnetic form factors involves taking matrix elements of the above currents. The full form factor can be written as a sum

$$
F^{B}\left(\mathbf{q}^{2}\right)=F_{i m p}^{B}+F_{g q \bar{q}}^{B}+F_{\pi q \bar{q}}^{B}+F_{\gamma \pi \pi}^{B}+F_{c o n f}^{B}+F_{\sigma}^{B}
$$

The different contributions to the magnetic moments $\mu^{B}=F^{B}\left(\mathbf{q}^{2}=0\right)$ for the octet baryons as calculated with the parameters of table 1 are given in table 3 .

First we recognize that the individual two-body currents contributions are rather large, especially the gluon and confinement part.

We find a slightly better overall agreement of our total result with the experimental values in comparison to the impulse result alone. We should stress, that none of the parameters is fitted to the baryon magnetic moments, but to the nucleon and $\Delta$-masses, and that these parameters allow to describe also the static deuteron properties [10 with reasonable accuracy. By varying the constituent quark masses (for example $m_{u}=324 \mathrm{MeV}, m_{u} / m_{s}=0.6$ ), we could improve our total result to get the aforementioned $5 \%$ agreement without giving up the good agreement for the baryon masses.

Because the isovector pion-pair and pionic currents largely cancel each other (for the proton we find $\mu_{\pi q \bar{q}}^{I V}=-0.29$ n.m. and $\mu_{\gamma \pi \pi}^{I V}=0.41$ n.m.), the pion cloud gives only a small correction for the nucleon magnetic moments ${ }^{2}$. To the charged $\Sigma^{ \pm}$only the small isoscalar pion-pair current contributes.

\footnotetext{
${ }^{2}$ We expect (in addition to a suppression due to the rather big Kaon mass) the same cancellation of the Kaon-pair current and the "Kaon-in-flight" contribution. This can be considered as further justification for our exclusion of Kaon and $\eta$ degrees of freedom in the present calculation.
} 
The second cancellation occurs between the gluon-, confinement- and $\sigma$-pair currents. This cancellation is clearly favored for a quark core radius $b_{N} \simeq 0.6 \mathrm{fm}$. This value for $b_{N}$ is also obtained from the variational principle $\frac{\partial M_{N}\left(b_{N}\right)}{\partial b_{N}}=0$ for $(0 s)^{3}$ shell model trial wave functions [2]. Furthermore, one can establish in this basis a relation between the neutron charge radius and the nucleon- $\Delta$ mass-splitting $<r^{2}>_{n}=-b_{N}^{2} \frac{M_{\Delta}-M_{N}}{M_{N}}$, which is fulfilled for $b_{N}=0.612 \mathrm{fm}$ [2]. The choice of $b_{N} \simeq 0.6 \mathrm{fm}$ is therefore the most consistent one in the restricted basis of $(0 s)^{3}$-states. Taking for example $b_{N}=0.5 \mathrm{fm}$ would completely spoil these cancellations, and we would find for the proton a magnetic moment of $\mu_{p} \simeq 5.6$ n.m. Another point to mention at this stage is, that the two-body current associated with a vector rather than a scalar confinement has the same sign as the gluon exchange current for all the octet baryons, i.e. the wrong sign to cancel the large gluon contribution. This has been observed before [2] for the case of the proton and neutron magnetic moments.

Let us now study the magnetic radii. The magnetic radii of hadrons provide valuable information about the inner structure of hadrons. In particular, they provide a further test of the gluon, pion, and scalar exchange currents discussed here. In table 4 , we present the magnetic radii

$$
\left\langle r^{2}\right\rangle^{B}=-\left.\frac{6}{\mu^{B}} \frac{\mathrm{d} F^{B}\left(\mathbf{q}^{2}\right)}{\mathrm{d} \mathbf{q}^{2}}\right|_{\mathbf{q}^{2}=0}=\left\langle r^{2}\right\rangle_{i m p}+\left\langle r^{2}\right\rangle_{g q \bar{q}}+\left\langle r^{2}\right\rangle_{\pi}+\left\langle r^{2}\right\rangle_{c o n f}+\left\langle r^{2}\right\rangle_{\sigma}
$$

First, we recognize, that the experimental proton and neutron magnetic radii can be nicely reproduced in our calculation. Throughout the whole octet, the net contribution of all the exchange current processes to the magnetic radii is quite small, however always negative (except for the $\Xi^{-}$). Thus the cancellation of two-body effects is also seen in the magnetic radii of the octet baryons. We observe that the strange baryons have a slightly smaller magnetic size than the proton and neutron. Unfortunately, there are no experimental data for comparison up to now. With the present experimental errors for the nucleon magnetic radii, it is difficult to find clear-cut evidence for exchange-current effects in these observables.

In this letter we have investigated the gauge invariant pion, gluon and scalar exchange 
current contributions to the magnetic moments and radii of the octet baryons. We have found substantial cancellations between different exchange current contributions. Because we neglect various other effects, such as small anomalous magnetic moments of the quarks, configuration mixing, relativistic effects beyond the two-body currents included here, one should not expect a perfect agreement with the experimental data. Nevertheless, we stress again, that our results are obtained without fitting any parameter to the magnetic moments and the magnetic radii, but using parameters fixed by the nucleon and $\Delta$-masses. It has been shown before, that the aforementioned cancellations between different exchange currents also occur, when improved wave functions (configuration mixing) are used [2].

Naturally the question arises: Are these cancellations between various two-body currents a coincidence, or is there a more fundamental reason why the cancellations are most pronounced just for $b_{N} \simeq 0.6 \mathrm{fm}=\frac{1}{329 \mathrm{MeV}} \simeq \frac{1}{m_{u}} ?$ In the latter case, the baryon magnetic moments could provide another argument for a universal nucleon harmonic oscillator length of $b_{N} \simeq 0.6 \mathrm{fm}$. This value gives a reasonable baryon size, and allows to describe the positive parity baryon spectrum and baryon electromagnetic observables with the same parameters.

In summary our main result is, that the - individually large — two-body contributions to the octet baryon magnetic moments tend to cancel each other, when the exchange currents are consistently evaluated with the Hamiltonian. Therefore our results offer a possible explanation for the surprisingly good agreement of the naïve additive quark model predictions with the experimental values. 


\section{References}

[1] Mesons in Nuclei II, edited by M. Rho and D. Wilkinson, North Holland, New-York, 1979

[2] A. Buchmann, E. Hernandez, and K. Yazaki, Phys.Lett.B269 (1991), 35;

A. Buchmann, E. Hernandez, and K. Yazaki, Nucl.Phys.A569 (1994), 661

[3] G. Karl, Phys.Rev.D45 (1992), 247

[4] F.E. Close, An Introduction to Quarks and Partons, Academic Press, London, 1979

[5] A. Buchmann, Y. Yamauchi, and A. Faessler, Nucl.Phys.A496 (1989), 621;

Phys.Lett.B225 (1989), 301; Progr.Part.Nucl.Phys.24, ed. A. Faessler, p. 333 (Pergamon Press, Oxford, 1990)

[6] for recent reviews see: W. Lucha, F.F. Schöberl, D. Gromes, Phys.Rep.200 (1991), 127;

M.M. Giannini, Rep.Prog.Phys.54 (1990), 453;

R.F. Alvarez-Estrada, F. Fernandez, J.L. Sanchez-Gomez and V. Vento, Lecture notes in physics 259 (Springer, Berlin, 1986)

[7] K. Shimizu, Phys.Lett.B148 (1984), 418; D. Robson, Phys.Rev.D35 (1985), 1029;

K. Maltman, Nucl.Phys.A446 (1985), 623;

F. Fernandez and E. Oset, Nucl.Phys.A455 (1986), 720

[8] I.T. Obukhovsky and A.M. Kusainov, Phys.Lett.B238 (1990), 142;

F. Fernandez, A. Valcarce, U. Straub and A. Faessler, J.Phys.G19 (1993), 2013

[9] A. DeRujula, H. Georgi, and S.L. Glashow, Phys.Rev.D12 (1975), 147

[10] A. Buchmann, Czech.J.Phys.45 (1995)

[11] A. Valcarce, A. Buchmann, F. Fernandez, and A. Faessler, Phys.Rev.C50 (1994), 2246; Phys.Rev.C51 (1995), 1480

[12] U. Vogl, M. Lutz, S. Klimt, W. Weise, Nucl.Phys.A516 (1990), 469

[13] N. Isgur and G. Karl, Phys.Rev.D18 (1978), 4187; Phys.Rev.D19 (1979), 2653

[14] Particle Data Group, M. Aguilar-Benitez et al., Phys.Rev.D50 (1994), 1173 


\section{Figure captions:}

Figure 1:(a) Impulse current, (b) pion-pair current, (c) pionic current, (d) gluon-pair current, and (e) scalar exchange current (confinement and $\sigma$-exchange). The black dots on the photon-quark and the photon-pion vertices indicate the finite electromagnetic size of the constituent quark and the pion, for which we take $r_{\gamma q}^{2}=r_{\gamma \pi}^{2}=0.36 \mathrm{fm}^{2}$. 
Georg Wagner et al., Figure 1,

"Magnetic Moments of the Octet ...", Phys.Lett.B

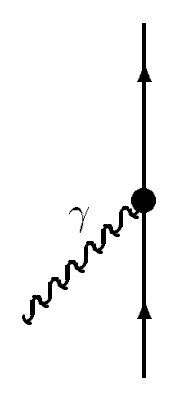

(a)

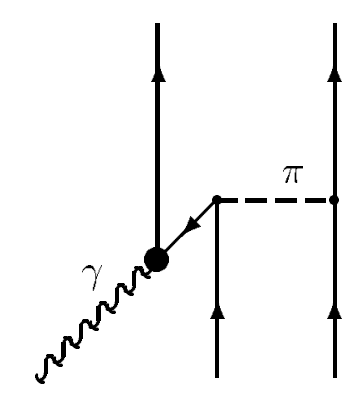

(b)

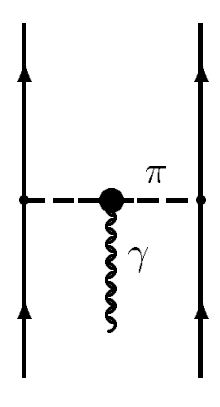

(c)

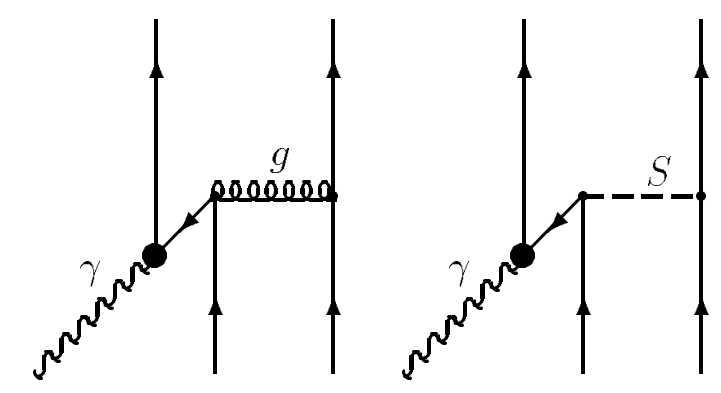

(d)

(e) 


\section{Tables:}

\begin{tabular}{|l|l|l|l|l|l|}
\hline$b_{N}$ & $m_{u}=m_{d}$ & $m_{u} / m_{s}$ & $\Lambda$ & $a_{c}$ & $\alpha_{s}$ \\
\hline \hline $0.613 \mathrm{fm}$ & $313 \mathrm{MeV}$ & 0.6 & $4.2 \mathrm{fm}^{-1}$ & $20.92 \mathrm{MeV} \mathrm{fm}^{-2}$ & 1.094 \\
\hline
\end{tabular}

Table 1: The parameters of Hamiltonian (1) for the present calculation

\begin{tabular}{|l||r|r|r|r|r|r||r|r|}
\hline & $\sum_{i} m_{i}$ & $E_{\text {kin }}$ & $V_{\text {conf }}$ & $V_{\text {gluon }}$ & $V_{\pi}$ & $V_{\sigma}$ & $m_{B}$ & $m_{\exp }[\mathbf{1 4}]$ \\
\hline \hline $\mathrm{p}, \mathrm{n}$ & 939 & 497 & 189 & -512 & -119 & -54 & 939 & 939 \\
\hline$\Delta$ & 939 & 497 & 189 & -314 & -24 & -54 & 1232 & 1232 \\
\hline$\Sigma$ & 1148 & 497 & 163 & -540 & -8 & -63 & 1197 & 1193 \\
\hline$\Lambda$ & 1148 & 497 & 163 & -561 & -71 & -63 & 1112 & 1116 \\
\hline$\Xi$ & 1356 & 497 & 138 & -613 & 0 & -74 & 1304 & 1318 \\
\hline
\end{tabular}

Table 2: The different contributions of Hamiltonian (1) to the baryon masses. All quantities are given in $[\mathrm{MeV}]$. The experimental values represent averages over particles with different charge. 


\begin{tabular}{|l||r|r|r|r|r||r|l|}
\hline octet & $\mu_{i m p}$ & $\mu_{g q \bar{q}}$ & $\mu_{\pi}$ & $\mu_{\text {conf }}$ & $\mu_{\sigma}$ & $\mu_{\text {tot }}$ & $\mu_{\exp }[14]$ \\
\hline \hline $\mathrm{p}$ & 3.000 & 0.598 & 0.149 & -1.205 & 0.347 & 2.889 & 2.793 \\
\hline $\mathrm{n}$ & -2.000 & -0.199 & -0.098 & 0.804 & -0.232 & -1.725 & -1.913 \\
\hline$\Sigma^{+}$ & 2.867 & 0.615 & 0.017 & -1.003 & 0.363 & 2.859 & $2.458 \pm .010$ \\
\hline$\Sigma^{-}$ & -1.133 & -0.361 & 0.017 & 0.444 & -0.156 & -1.190 & $-1.160 \pm .025$ \\
\hline$\Xi^{0}$ & -1.467 & -0.040 & 0.000 & 0.349 & -0.175 & -1.333 & $-1.250 \pm .014$ \\
\hline$\Xi^{-}$ & -0.467 & -0.219 & 0.000 & 0.028 & -0.031 & -0.689 & $-0.651 \pm .003$ \\
\hline$\Lambda$ & -0.600 & -0.018 & 0.000 & 0.116 & -0.052 & -0.554 & $-0.613 \pm .004$ \\
\hline$\Sigma^{0} \rightarrow \Lambda$ & 1.732 & 0.154 & 0.000 & -0.278 & 0.125 & 1.733 & $1.61 \pm .08$ \\
\hline
\end{tabular}

Table 3: Different contributions to the octet baryon magnetic moments in comparison to the experimental values. The first column $\mu_{i m p}$ shows the impulse result, to be compared with the total result $\left(\mu_{t o t}\right)$ and the experimental values in the last column. The second, third, forth and fifth column contain respectively the exchange current contributions due to the gluon, the pion (pion-pair and pionic part), the confinement and the $\sigma$-exchange. All quantities are given in n.m. $\mu_{N}=\frac{e}{2 M_{P}}$. The last line contains the transition magnetic moment for the decay $\Sigma^{0} \rightarrow \Lambda$.

\begin{tabular}{|l||r|r|r|r|r||c|c|}
\hline octet & $\left\langle r^{2}\right\rangle_{\text {imp }}$ & $\left\langle r^{2}\right\rangle_{g q \bar{q}}$ & $\left\langle r^{2}\right\rangle_{\pi}$ & $\left\langle r^{2}\right\rangle_{\text {conf }}$ & $\left\langle r^{2}\right\rangle_{\sigma}$ & $\sqrt{\left|\left\langle r^{2}\right\rangle_{\text {tot }}\right|}$ & $r_{\text {exp }}$ [2] \\
\hline \hline $\mathrm{p}$ & 0.764 & 0.117 & 0.132 & -0.385 & 0.043 & 0.819 & $0.858 \pm 0.056$ \\
\hline $\mathrm{n}$ & 0.853 & 0.065 & 0.203 & -0.430 & 0.048 & 0.860 & $0.876 \pm 0.070$ \\
\hline$\Sigma^{+}$ & 0.702 & 0.116 & 0.003 & -0.303 & 0.046 & 0.751 & \\
\hline$\Sigma^{-}$ & 0.683 & 0.164 & -0.008 & -0.324 & 0.047 & 0.750 & \\
\hline$\Xi^{0}$ & 0.706 & 0.015 & 0.000 & -0.206 & 0.047 & 0.750 & \\
\hline$\Xi^{-}$ & 0.370 & 0.162 & 0.000 & -0.026 & 0.016 & 0.723 & \\
\hline$\Lambda$ & 0.763 & 0.017 & 0.000 & -0.172 & 0.034 & 0.801 & \\
\hline$\Sigma^{0} \rightarrow \Lambda$ & 0.704 & 0.048 & 0.000 & -0.206 & 0.026 & 0.756 & \\
\hline
\end{tabular}

Table 4: Different contributions to the octet baryon magnetic radii, and comparison with the experimentally available data. The notations are analogous to the ones in table 3. All quantities are given in $\left[\mathrm{fm}^{2}\right]$, in the last two columns in $[\mathrm{fm}]$. 